\title{
A Controvérsia Entre os Métodos de Análise de Projetos de Referência em Arquitetura e o Ensino de Projeto Performativo
}

\author{
The Controversy Between Reference Projects Analysis Methods in Architecture and the Performative \\ Design Teaching
}

\author{
Leandro Silva Leite \\ Universidade Federal de Santa Catarina, Brasil \\ arqlsleite@gmail.com \\ Alice Theresinha Cybis Pereira \\ Universidade Federal de Santa Catarina, Brasil \\ acybis@gmail.com
}

\begin{abstract}
The architecture of the digital age yields complex shapes exemplars which once divulged on the internet, they become architectonic references. This article aims to discuss the influence of the reference projects analysis process (APR) in the projects generation within the current teaching. A comparative assessment of undergraduate final project has been carried out so as to identify the APR methods applied. From the application of the theory - Social Construction of Technologies (SCOT), the 05 facts that became evident show the controversy between the APR teaching processes and the interdisciplinary knowledge and abilities needed for the generation of complex and/or performative shapes.
\end{abstract}

Keywords: Reference projects analysis; Architecture projects teaching; Performative project.

\section{Introdução}

Busca-se, no presente artigo, o entendimento da relação entre a qualidade e estruturação do processo de análise de projetos de referência em arquitetura, e o potencial de desenvolvimento de conhecimentos e habilidades necessários para a compreensão de campos de saber interdisciplinares inerentes ao processo de projeto performativo.

É composto por uma breve revisão bibliográfica com o objetivo de descrever o conceito de arquitetura performativa e a importância dos processos de simulação, Kolarevic (2005) e Malkawi (2005), e contrapor autores sobre confronto entre utopia e pragmatismo, Umakoshi e Gonçalves (2009) e Roaf (2009), no intuito de compreender a relação entre o os processos de geração da forma e sua viabilidade construtiva, Andrade (2012) e Arantes (2012).

A presente pesquisa é composta de duas etapas, faz-se na primeira etapa, um estudo comparativo entre os Trabalhos de Conclusão no intuito de identificar os métodos de análise de projeto de referência (APR) como o objetivo de contextualizar os resultados obtidos na segunda etapa, composta por um processo de investigação da aprendizagem de projeto de arquitetura por interdisciplinaridade na realidade brasileira com base em uma visão socioconstrutivista pela aplicação da teoria - Social Construction of Technologies (SCOT), Bijker (1987), tendo como referência da interpretação da teoria o trabalho de Firmino (2007).

\section{O conflito entre utopia e pragmatismo}

A busca por soluções para a melhora no desempenho ambiental das edificações, desde a crise petrolífera na década de 1970, torna-se um fator fundamental no processo de concepção arquitetônico. Mais do que uma discussão de forma/função, menos ou mais, complexo ou contraditório, somos uma geração responsável por desenvolver uma arquitetura que utilize os avanços tecnológicos em prol da sustentabilidade dos recursos energéticos e ambientais.

Para Umakoshi e Gonçalves (2009) o desafio de uma geração de uma arquitetura de melhor desempenho ambiental está na atuação de arquitetos que explorem o potencial da forma, das relações espaciais, dos componentes construtivos, junto com uma atitude visionária que questione e desafie os modelos vigentes.

Para Roaf (2009) as escolas de arquitetura estão dominadas por "entretenimentos arquitetônicos" e responsabiliza o corpo docente pelo ensino das mesmas, principalmente pela íntima relação destes com as técnicas de simulação digital. Para validar seu raciocínio cita Peter Cook, diretor da Barlett School of Architecture, um dos fundadores do grupo Archigram e ele próprio projetista de blobs, o qual estaria muito preocupado com a crescente obscuridade do trabalho dos alunos e que no futuro iria 
se certificar pessoalmente de que o trabalho seja melhor explicado e descrito.

De acordo com os relatos acima nos deparamos com duas visões distintas quanto aos desafios a serem enfrentados pelas gerações na busca pela concepção de edificações de melhor desempenho ambiental. Uma visão alternativa pode estar no trabalho desenvolvido pelo grupo Bjarke Ingels (BIG) (2011), para o qual a arquitetura parece encurralada entre duas frentes igualmente estéreis: a ingenuidade utópica ou o pragmatismo petrificante. 0 ideal do grupo é não ter de escolher um ou outro, mas sim trabalhar nos férteis terrenos comuns entre os dois opostos, em uma arquitetura por eles descrita como "utópica-pragmática" e que tem por objetivo prático a criação de locais perfeitos a nível social, econômico e ambiental.

Seria possível uma visão de vanguarda onde o conhecimento especializado teria como ferramenta de auxílio para visualização, simulação e concepção da forma e das relações espaciais as técnicas gráficas e digitais e como objetivo principal a busca por um projeto arquitetônico de comprovado desempenho?

\section{Projeto perfomativo entre a geração e a renda da forma}

Os ideais de vanguarda descritos por Montaner (2007), a confiança de que existe uma racionalização intrínseca no mundo da tecnologia e da ciência; ideia do progresso ilimitado e confiança em que os novos materiais e as novas disponibilidades tecnológicas permitiriam superar todos os condicionantes da arquitetura tradicional podem ser relacionadas com a arquitetura performativa descrita por Kolarevic (2005) como tendo uma capacidade de responder às mudanças das condições sociais, culturais e tecnológicas por perpetuamente reformatar-se como um índice, bem como um mediador de (ou uma interface para) padrões culturais emergentes.

Um dos elementos fundamentais da arquitetura performativa descritos por Malkawi (2005) é o processo de simulação, o autor relata a importância de automatizar alguns dos processos de simulação, a fim de que o projeto possa utilizar critérios de desempenho térmico e ventilação como meio de gerar alternativas de design.

Em uma análise crítica, para Arantes (2012) nas últimas duas décadas a arquitetura embarcou definitivamente no universo midiático das logomarcas, trata-se de uma arquitetura que circula como imagem e, por isso, nasce figura de si mesma, pela redução da experiência arquitetônica à pura visualidade, resultado da busca incessante pelo ineditismo e pelo que o autor chamou de "renda da forma", uma renda monopolística intrínseca à sua forma arquitetônica única e espetacular, capazes de valorizar os investimentos e, consequentemente, as cidades que os disputam.

Contudo, Andrade (2012) demonstra pela criação de uma estrutura conceitual e pela aplicação de modelo performativo em experiência de ensino em ateliê que o Projeto Performativo pode aliar a estética por meio da busca de uma arquitetura que seja capaz de atrelar geração da forma arquitetônica à eficiência energética e sustentabilidade.

\section{Identificação dos Métodos de Análise de Projetos de Referência}

No intuito de compreender dentro do processo de projeto como na etapa de análise ${ }^{1}$, Lawson (2011), os métodos de análise gráfica e textual de projetos de referência em arquitetura são utilizados, fez-se um estudo comparativo entre os quatro trabalhos de conclusão pertencentes à pesquisa. Buscou-se identificar e classificar os métodos de APR utilizados a fim de contextualizar os resultados obtidos pela aplicação da SCOT. Como resultado foi possível identificar 05 métodos de APR:

APR - 01 (Figura 1) - Análise Gráfica de imagens.

APR - 02 (Figura 1) - Descrição textual com base em publicações (sites, artigos, revistas, livros).

APR - 03 (Figura 1) - Descrição visual de soluções técnicas.

APR - 04 (Figura 1) - Quadro de análise descritiva com base em publicações.

APR - 05 (Figura 2) - Análise gráfica com base em modelo digital 3D.

APR - 06 (Figura 2) - Quadro de análise comparativa entre projetos de referência com base em publicações.

Da análise comparativa é possível inferir que existe uma relação direta entre a APR utilizada e a influência nos conhecimentos obtidos de concepção da forma em projeto de arquitetura. Este fato é evidenciado pelo exemplar de 2009 (Figura 1), o qual ao realizar APR se apropria dos conhecimentos de programa e identidade formal do correlato, contudo ao realizar APR não aprofundada do sistema estrutural e materiais utilizados acaba replicando na especificação do projeto de TC soluções construtivas sem uma análise crítica. Como a realizada por Arantes (2012) o qual relata que o projeto da Cada da Música de Rem Koolhaas vencera $o$ concurso sem definir qual seria o material que constituiria a pela superficial do prisma irregular. "A desconsideração de Koolhaas pelo material (matéria mais trabalho) é gritante na fachada do edifício...Os construtores foram submetidos a um esforço exaustivo para dispor as fôrmas inclinadas segundo o grid traçado em computador"(p.90).

\footnotetext{
${ }^{1}$ A análise envolve a investigação das relações na busca de algum padrão nas informações disponíveis e a classificação dos objetivos. A análise é o ordenamento e a estruturação do problema.(Lawson, 2011, p. 45)
} 


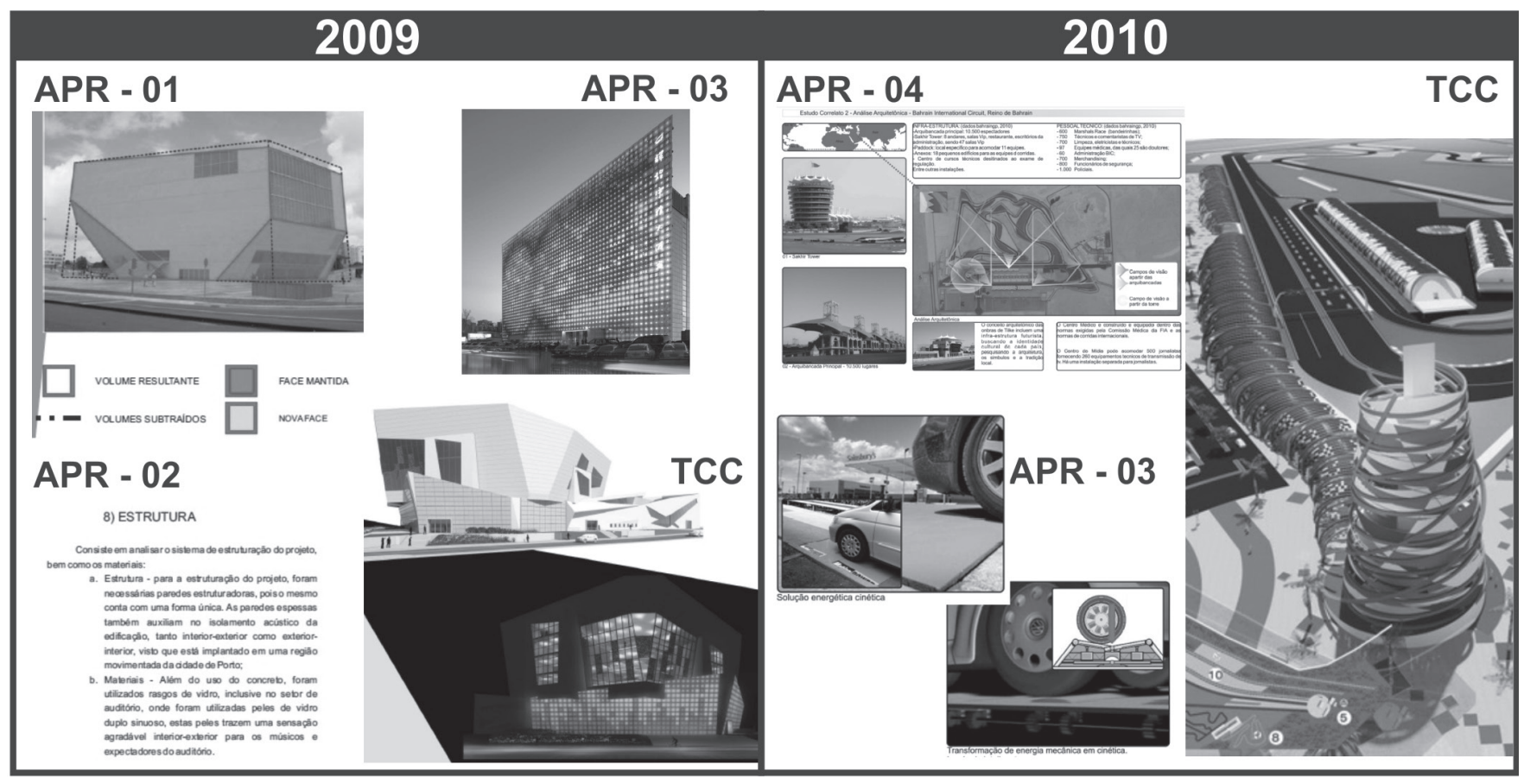

Figura 1: Exemplar 2009 TCC - Casa da Música e Exemplar 2010. TCC - Autódromo Internacional.

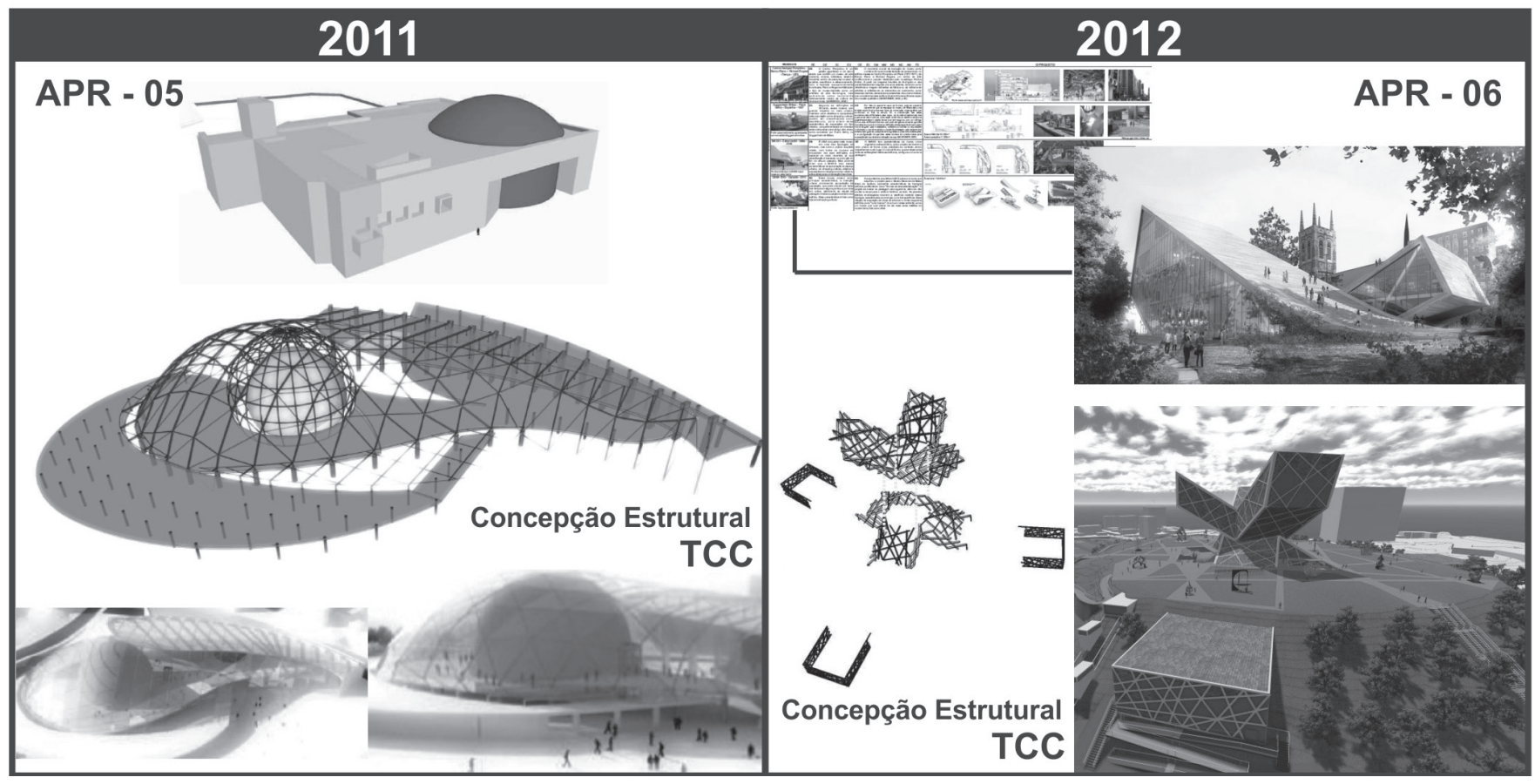

Figura 2: Exemplar 2011 TCC - Centro de Realidade Virtual e Exemplar 2012 TCC - Museo de Arte Contemporânea.

O exemplar de 2010 (Figura 1) demonstra que a descrição detalhada de um projeto de referência, bem como a comparação entre projetos é uma técnica fundamental principalmente em projetos com alto grau de complexidade funcional, seja pelo uso inerente, seja pelo desconhecimento prévio de suas características por parte do aluno. Contudo, para que os detalhamentos técnicos sejam integrados ao projeto é necessário que o processo de descrição visual seja complementado por multimétodos de APR.
O exemplar de 2011 (Figura 2) evidencia a relação direta entre o exercício de análise gráfica digital tridimensional e o desenvolvimento da capacidade e habilidade de avaliação de conhecimentos interdisciplinares (concepção da forma e concepção estrutural) em um processo de construção virtual em 3D.

O exemplar de 2012 (Figura 2) demonstra a relação entre a qualidade da descrição do processo de geração da forma realizado 
pelo BIG como instrumento de aprendizagem por parte do aluno para a compreensão de estudos de conhecimentos interdisciplinares (concepção da forma e concepção estrutural) também em um processo de construção virtual em 3D. Contudo, tem-se o indício de que análises complementares realizadas pelo próprio aluno são fundamentais para que não ocorra uma excessiva similaridade com o projeto de referência selecionado.

Como conclusão da análise comparativa é possível inferir que no processo de APR tem similar importância a utilização de multimétodos de análise, desde o textual aprofundado à análise gráfica digital em modelo 3D. O qual é possível ser identificado como processo de análise multimétodos de projetos de referência (AMPR).

\section{Resultados Parciais da SCOT}

Para a aplicação da SCOT, selecionaram-se quatro Trabalhos de Conclusão de Curso de um exemplar de Curso de Graduação em Arquitetura e Urbanismo no Estado de Santa Catarina, Brasil, no período entre 2009 e 2012, com base em dois critérios de projeto: utilização de processo estruturado de APR em arquitetura e utilização de integração interdisciplinar de critérios de sustentabilidade e/ou eficiência energética na concepção das propostas. Como técnica de investigação, realizou-se entrevista semiestruturada com os principais atores responsáveis pelo processo Trabalhos de Conclusão de Curso (alunos, coordenador de curso e coordenadores de TC). As entrevistas foram transcritas e o conteúdo foi tratado por método de análise de conteúdo, Bardin (2002). A partir dos resultados obtidos, identificaram-se as flexibilidades interpretativas, fechamento e estruturações que configuram a visão socioconstrutivista do processo de aprendizagem de projeto de arquitetura.

Como resultados da aplicação da pesquisa, foram encontradas quatro controvérsias principais em relação ao ensino de projeto interdisciplinar de arquitetura e as possibilidades de ensino de projeto performativo. Uma destas controvérsias tem relação direta com a influência que o livre acesso às mídias digitais (sites, blogs e redes sociais) tem sobre a APR:

Controvérsia: A arquitetura na era digital produz exemplares de formas complexas, os quais são divulgados e analisados por meio de mídias digitais e se tornam projetos de referência em arquitetura, mas o processo de APR não segue uma estruturação multimétodos adaptada para a busca de um entendimento dos conceitos interdisciplinares presentes na concepção de projetos performativos.

Da análise da flexibilidade interpretativa, a partir dos relatos dos atores envolvidos no processo, pode se inferir os seguintes fatos presentes na realidade do ensino de projeto de arquitetura:
Fato 01 - Os Coordenadores demonstraram controvérsia com relação à fase em que as ferramentas digitais deveriam ser inseridas no ensino de projeto, os relatos oscilaram entre o início e o meio do curso. Pode-se inferir que esta divergência está ligada ao fato de a maioria relacionar a utilização de ferramentas digitais com o processo de representação gráfica, esta dedução se dá pelo relato quanto ao receio do aluno não desenvolver conhecimentos e habilidades manuais, principalmente noções básicas de espacialidade, proporção e escala.

Fato 02 - Todos os alunos relataram utilizar ferramentas digitais durante o processo de projeto, a maioria desde a APR. Contudo, relatam que as ideias iniciais são lançadas de forma manual e após definido o conceito e/ou partido todo o restante do processo é realizado em ferramentas digitais em processo de construção virtual 3D e com retorno ao manual quando necessário.

Fato 03 - Todos os alunos relataram iniciar a pesquisa de referenciais de arquitetura por páginas de busca na internet (Google), fato que é indício da força que os projetos divulgados por mídias digitais possuem enquanto influência no processo de aprendizagem de projeto de arquitetura. Contudo, todos os coordenadores relataram não ter utilizado no período de 2009 a 2012, Ambientes Virtuais de Aprendizagem para o ensino e/ou avaliação dos referenciais viabilizados via mídias digitais. Este fato é uma possível evidência de que o modo como as informações obtidas via mídias digitais sobre os referenciais de arquitetura são analisadas não possui uma estrutura específica de ensino/aprendizado.

Fato 04 - É consenso entre os entrevistados a importância do processo de análise e síntese, como descritos por Lawson (2011), ser realizado com base em ferramentas 3D, mas pouco é mencionado a respeito do processo de avaliação por simulação. Todos os entrevistados relataram ter pouco ou nenhum conhecimento sobre os termos: arquitetura performativa, arquitetura paramétrica, Building Information Modeling e Prototipagem Rápida. Este fato é coincidente com o relato sobre experiência de ensino de projeto performativo realizado por Andrade (2012, p.311) "Softwares de Autoria BIM, como o Revit Architecture ou o ArchiCAD são pouco conhecidos pela turma e os usuários têm pouca experiência".

Fato 05 - É consenso entre os alunos a relação direta entre o conhecimento e habilidade em ferramentas digitais para construção virtual 3D e a capacidade para gerar formas de geometria complexa, com agilidade e precisão. Contudo, a maioria dos alunos relatou ter obtido esta habilidade fora do ambiente acadêmico (cursos ou estágios). $\mathrm{E}$ ter tido dificuldades no desenvolvimento do TC por divergência entre o método de avaliação baseado em representação gráfica 2D exigido pelos critérios de entrega intermediários e o processo de construção virtual 3D. Pode-se inferir por este fato que o tempo que poderia 
ser utilizado para avaliações de desempenho acaba sendo gasto para adequar o processo de construção virtual 3D ao processo de avaliação convencional.

O fato 01 demonstra que existe insegurança quanto à inserção de ferramentas digitais no ensino de projeto de arquitetura, o fato 02 esclarece que a forma adequada para os alunos é a variação entre métodos manuais e digitais, em acordo com a teoria apresentada por Andrade (2012). O fato 03 evidencia que as mídias digitais são elementos de forte influência na seleção dos referenciais de arquitetura e de que é necessário o desenvolvimento de métodos estruturado de ensino/aprendizagem de AMPR.

Os fatos 04 e 05 indicam que a estrutura necessária para o ensino/aprendizagem de projeto performativo possui além da falta de modelos de AMPR adequados, mais deficiências a serem superadas como: a conscientização da importância da concepção de projetos com base na construção virtual 3D; a necessidade de instrumentalizar os alunos para a utilização de ferramentas de parametrização digital; a importância de inserir as simulações de desempenho dentro da estrutura de avaliação dos projetos de modo a valorizar a qualidade da performance da forma e não somente da qualidade da imagem que a mesma gera.

\section{Considerações Finais}

Com base nos relatos apresentados é possível reconhecer como o fechamento da visão socioconstrutivista sobre ensino de projeto de arquitetura e a influência de mídias digitais na definição e análise de referenciais de arquitetura como: 0 processo de inserção de ferramentas digitais no ensino de projeto de arquitetura não abrange com eficiência a etapa de análise de referenciais, principalmente em busca de entendimento dos conhecimentos interdisciplinares que condicionaram a concepção da forma.

E a estrutura desta visão pode ser descrita como: A seleção de referenciais de arquitetura é realizada via mídias digitais em função da imagem do projeto e a sua influência está diretamente ligada ao processo de lançamento de partido arquitetônico com base na valorização da "Idea" do arquiteto.

Romper com esta estrutura estabelecida e abrir novos caminhos para a passagem de um processo utópico e/ou idealizado para um processo "utópico-pragmático", a iniciar pela sistematização de processos de AMPR adequados ao ensino de projeto de arquitetura de formas complexas e/ou performativos, é fato de evidente importância para a implementação do ensino de projeto performativo no Brasil.

\section{Agradecimentos}

CAPES pelo financiamento desta pesquisa.

\section{Referências}

Andrade, Max L. V. Xavier de.(2012). Projeto Performativo na prática arquitetônica recente: estrutura conceitual. Campinas. SP.

Arantes, Pedro F. (2012). Arquitetura na era digital-financeira: desenho, canteiro e renda da forma. (1a Ed). São Paulo: Editora 34.

Bardin. Laurence. (2002). Análise de Conteúdo. Trad. Luís Antero Reto e Augusto Pinheiro. Lisboa: Edições 70.

Bijker, W.(1987). Of Bicycles, Bakelites, and Bulbs: Toward a Theory of Sociotechnical Change. Cambridge. MA. MIT Press.

Bjarke Ingels Group (BIG). (2011). Yes is More - um arqui-comic sobre a evolução arquitetônica. Editora Taschen.

Firmino, Rodrigo J. (2007). A cidade ampliada e o planejamento local no Brasil. A construção social do desenvolvimento urbano-tecnológico no interior de São Paulo. Universidade de São Paulo. São Paulo.

Kolarevic, Branko. (2005). Towards the performative in architecture. In: Performative architecture beyond instrumentality. Malkawi, Ali M.; Kolarevic, Branko. Londres, U.K.Spon Press.

Lawson, Bryan. (2011). Como arquitetos e designers pensam. São Paulo: Oficina de textos.

Malkawi, Ali M.(2005). Performance Simulation: Research and tools. In: Performative architecture beyond instrumentality. Malkawi, Ali M.; Kolarevic, Branko. Londres, U.K.Spon Press.

Montaner, Josep-Maria.(2007). Depois do movimento moderno. Ed. Gustavo Gili.

Roaf, Sue. (2009). A adaptação de edificações e cidades à mudanças climáticas. Porto Alegre: Ed. Bookman.

Umakoshi, Erica M.; Gonçalves, J Umakoshi, Erica M.; Gonçalves, Joana C. (2009). A utopia do edifício alto "verde"e a criação de uma nova geração de ícones do desempenho ambiental. Programa do Programa de Pós-Graduação em Arquitetura e Urbanismo da FAUUSP. 